\title{
Estudios ornitológicos en Loreto, Perú: notas sobre su historia
}

\author{
Ornithological studies in Loreto, Peru: Notes on its history
}

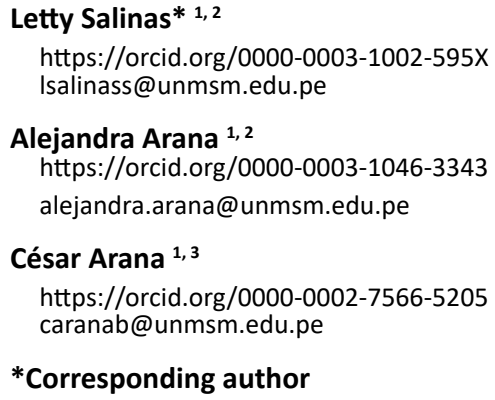

1 Universidad Nacional Mayor de San Marcos, Facultad de Ciencias Biológicas, Lima, Perú.

2 Universidad Nacional Mayor de San Marcos, Museo de Historia Natural, Departamento de Ornitología, Lima, Perú.

3 Universidad Nacional Mayor de San Marcos, Museo de Historia Natural, Departamento de Ecología, Lima, Perú.

\section{Citación}

Salinas L, Arana A, Arana C. 2021. Estudios ornitológicos en Loreto: notas sobre su historia. Revista peruana de biología 28(especial): e21916 001- 010 (Diciembre 2021). doi: http://dx.doi.org/10.15381/rpb.v28iespecial.21916

\section{Presentado: $\quad 09 / 02 / 2021$

Aceptado: $\quad 10 / 08 / 2021$ \\ Publicado online: 30/12/2021}

Editor:

Leonardo Romero

\section{Resumen}

En 1542 Orellana llega al Amazonas e inicia una historia de exploración continua de Loreto. Sin embargo, los estudios de las aves de este departamento recién empiezan en el siglo XIX con las exploraciones de Johann Baptist von Spix, Johann Jakob Von Tschudi, Francis-Louis de Castelnau, Emile Deville, Edward Bartlett, John Hauxwell y Henry Walter Bates quienes colectan para museos europeos y estadounidenses. En 1850 llega Antonio Raimondi al Perú incorporándose a la Universidad Nacional Mayor de San Marcos e iniciando los estudios nacionales de aves loretanas en 1859 y 1869. Raimondi colaboró activamente con Władysław Taczanowski, asistidos por Konstanty Jelski y Jan Stolzmann, cuyas colectas en Loreto fueran usadas en Ornithologie du Pérou, primer tratado de la avifauna peruana, conservándose algunos especímenes en el Museo de Historia Natural de la UNMSM (MHN). A inicios del siglo XX, Malcolm Anderson y Wilfred Osgood y posteriormente los hermanos Olalla colectaron para museos estadounidenses, marcando el inicio del predominio norteamericano en la ornitología en Perú. El investigador peruano Javier Ortiz de la Puente, primer jefe de la Sección Aves del MHN, realizó colectas en Loreto en 1948 y 1952, antes de su prematura muerte. María Koepcke, siguiente responsable de la Sección Aves del MHN, colecta en Pacaya-Samiria en 1967. John Patton O'Neill visita Loreto en 1963, iniciándose un interés que lo llevaría a impulsar la ornitología en Perú por varias décadas. La investigación continua de la avifauna loretana convirtió a esta región en un punto clave para entender los patrones de diversificación de las aves amazónicas.

\section{Abstract}

In 1542 Orellana arrived in the Amazon and began a history of continuous exploration of Loreto. However, the birds' study of Loreto began in the 19th century with the explorations of Johann Baptist von Spix, Johann Jakob von Tschudi, Francis-Louis de Castelnau, Emile Deville, Edward Bartlett, John Hauxwell and Henry Walter Bates, who collected for European and American museums. In 1850 Antonio Raimondi arrived in Peru, joining the Universidad Nacional Mayor de San Marcos, and starting national studies of birds of Loreto in 1859 and 1869. Raimondi actively collaborated with Władysław Taczanowski, assisted by Konstanty Jelski and Jan Stolzmann, whose collections in Loreto were used in Ornithologie du Pérou, the first treatise on Peruvian avifauna. Some of these specimens are preserved in the Museo de Historia Natural of the UNMSM (MHN). At the beginning of the 20th century, Malcolm Anderson and Wilfred Osgood and later the Olalla brothers collected for American museums, marking the beginning of American predominance in ornithology in Peru. The Peruvian researcher Javier Ortiz de la Puente, the first head of the Bird Section of the MHN, collected in Loreto in 1948 and 1952, before his unfortunate death. María Koepcke, next head of the MHN Bird Section, collected in Pacaya-Samiria in 1967. John Patton O'Neill visited Loreto in 1963, initiating an interest that would lead him to promote ornithology in Peru for several decades. The continuous research of Loreto's avifauna turned this region into a key point to understand the diversification patterns of Amazonian birds.

Palabras clave:

Historia de la ornitología, Perú, Amazonia, colecciones científicas, expediciones.

Keywords:

History of ornithology, Peru, Amazonia, scientific collections, expeditions. 


\section{Introducción}

Desde la llegada de los españoles al Perú, la Amazonia se adueñó del imaginario occidental. La búsqueda de "El Dorado", el "País de la canela" y hasta de las legendarias "Amazonas" impulsaron una exploración temprana del oriente peruano que llevó al "descubrimiento" del río Amazonas en 1542 por Francisco Orellana (Espinoza Soriano 2007). La impactante belleza de la naturaleza de esta región maravilló de tal modo a los occidentales que incluso llevó a Antonio de León Pinelo entre 1647 y 1650 a postular su teoría de la ubicación del Edén en esta región (Fig.1, León Pinelo 1943).

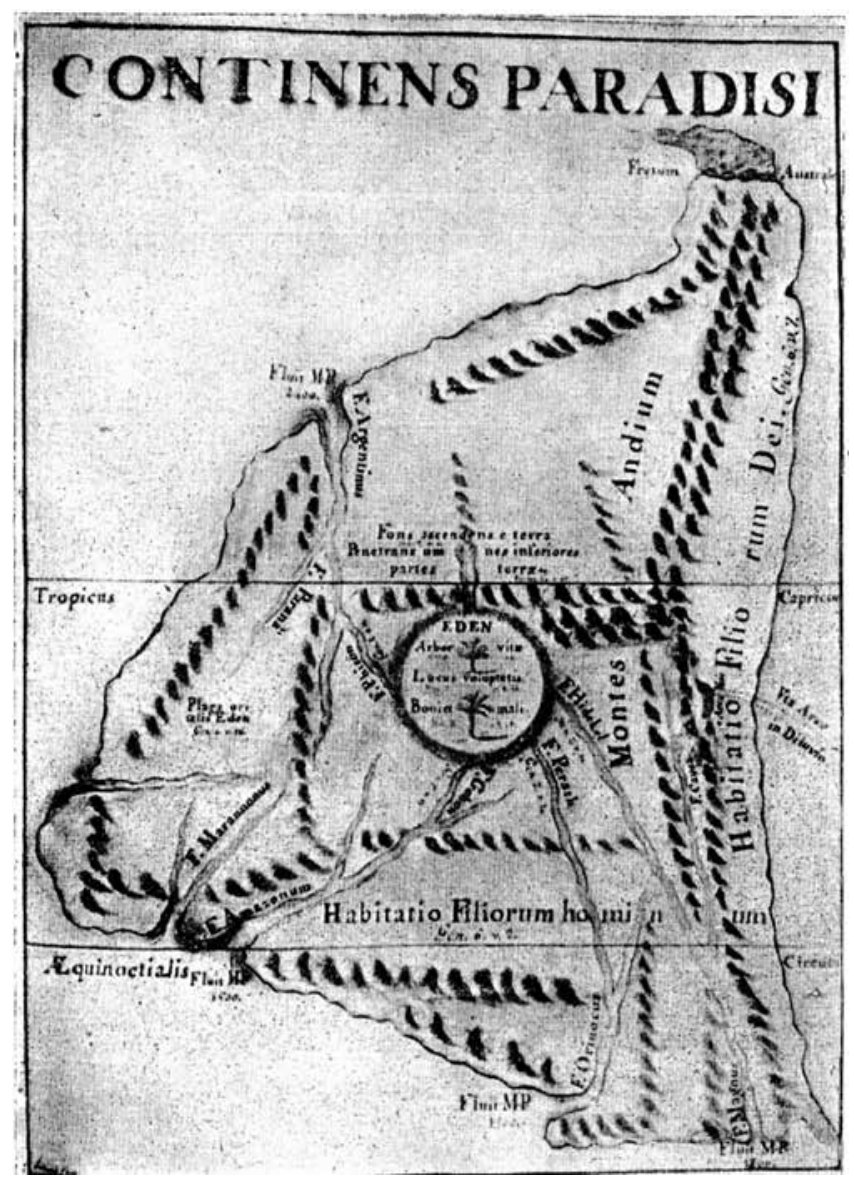

Figura 1. Mapa del Paraíso en la Amazonia según Antonio de León Pinelo (foja 126 del manuscrito de 1647-1650). Modificado de: Scott (2010).

Sin embargo, a diferencia del aporte al conocimiento ornitológico que brindó el Obispo Martínez de Compañón en el Obispado de Trujillo durante el Virreinato de Perú que incluyó también algunas especies de origen selvático (Macera et al. 1997), no ocurrió ningún aporte similar al conocimiento de la avifauna de la Amazonia durante el periodo de la Real Audiencia de Quito que comprendió el territorio de Loreto desde 1563 a 1802 (Real Cédula del 15 de julio de 1802), ni en el periodo inmediato cuando el Área de Loreto fue parte de la Comandancia y Capitanía de Maynas perteneciente al Virreinato de Perú con capital en la antigua ciudad de Moyobamba (Pons Muzzo 1962). En el virreinato además de Moyobamba fue muy conocida la ciudad de Yurimaguas que fue fundada en
1709 al pie del río Huallaga. Ocurrida la independencia del Perú en 1821 se fundan en las siguientes décadas, primero la ciudad de Nauta (1830) y posteriormente Iquitos (1866) que fuera declarada luego la capital de Loreto (1897), ya habiéndose separado esta región de lo que hoy es San Martín en 1853.

Antes de la fundación de Iquitos, ya se destacaba esta ciudad en los mapas de Perú (Paz Soldán 1865) y su posición relevante en la navegación por el Amazonas. En especial durante el "boom" cauchero alrededor de 1868 y 1907, la región Loreto se convierte en un área de interés mundial, no sólo comercial, sino también científico, al empezar a tener mayor conocimiento de las rutas de acceso a su territorio, a través del trabajo de la Comisión Hidrográfica del Amazonas dirigida por John Tucker desde 1867, que brindó amplia precisión al Mapa geográfico del río Amazonas realizado por Samuel Fritz en 1707 (Espinoza Soriano 2007).

El presente estudio resume las principales expediciones, colectas y estudios sobre aves realizados en la región Loreto, en especial los anteriores al siglo XX y que permitieron iniciar la documentación de su avifauna $y$ captar la atención de los científicos modernos sobre esta especialmente diversa área del Perú.

\section{Material y métodos}

Para el presente trabajo, la recopilación de la información histórica de los estudios ornitológicos en Loreto se inició con la revisión de los especímenes correspondientes a Loreto de la Colección Ornitológica del Museo de Historia Natural de la Universidad Nacional Mayor de San Marcos. Posteriormente, se buscó información en las bases de datos de otras colecciones científicas como las del Smithsonian National Museum of Natural History (NMNH 2020), del American Museum of Natural History (AMNH 2020), o las reunidas en la Global Biodiversity Information Facility (GBIF 2020), Integrated Digitized Biocollections (iDigBio 2020) y VertNet (VertNet 2020). Se incluyeron también datos de los inventarios de colecciones científicas publicados (Sharpe 1874, Brabourne \& Chubb 1917, Hastert 1918, Zimmer 1924, Zimmer 1937, van den Hoek Ostende et al. 1997, LeCroy \& Sloss 2000, Roselaar 2003, Lecroy 2005, Mlíkovský 2009, Lecroy 2012).

A esta información se sumaron los datos de publicaciones resultantes de la búsqueda bibliográfica utilizando los buscadores Google, Google Scholar y la base de datos Scopus, con diferentes combinaciones de las palabras clave: Loreto, birds, Amazonia, Aves, río Tigre, río Nanay, río Napo, río Amazonas, río Ucayali, río Marañón, río Huallaga, río Pastaza, río Corrientes, río Putumayo, río Yavarí, avifauna, Pebas, Yaguas, Iquitos, Yurimaguas, Nauta y Requena. En especial fue valiosa la revisión de la Biodiversity Heritage Library (BHL 2020) que permitió registrar información de las expediciones en las que se colectaron especímenes de valor histórico (anteriores al siglo XX) (Sclater 1857b; Sclater \& Salvin 1867; Sharpe 1874; Bartlett 1882; Taczanowski 1884a, 1884b, 1886). 


\section{Resultados y discusión}

Primeras expediciones y colectas para museos extranjeros. - La investigación ornitológica en Perú durante las primeras décadas de 1800 fue realizada principalmente por investigadores cuyas colectas fueron depositadas en instituciones europeas y norteamericanas (Sharpe 1874), principalmente en el British Museum (Natural History) - ahora Natural History Museum -, el Muséum national d'Histoire naturelle de París, los Museum of Vassar College y American Museum of Natural History en New York, Maidstone Museum en Reino Unido y el Museum and Institute of Zoology, Polish Academy of Sciences en Varsovia. Esta primera etapa estuvo fuertemente marcada por el descubrimiento de nuevas especies y subespecies de aves.

Es así como Loreto fue explorado por algunos de los más notables colectores de inicios y mediados del siglo XIX, como Johann Baptist von Spix en Tabatinga en el curso del Amazonas en la frontera entre el Perú y Brasil, en el hábitat llamado por él "Amazonum" entre 1817 y 1820 (Spix 1824). Loreto también fue explorado por Johann Jakob Von Tschudi quien colectó aves en los "bosques del Perú oriental, bosques vírgenes, bosques espesos, región boscosa oriental", incluyendo Yanayacu, posiblemente hacia el final de su exploración en el Perú entre 1838 y 1842 (Tschudi 1844-1846a, 1966; Taczanowski 1884a, 1884b), así como por Francis-Louis de Castelnau quien colectó en Misión de Sarayacu, Pampa del Sacramento, Ucayali, Nauta, Pebas y los ríos Amazonas y Yavarí, junto con Emile Deville (de Castelnau 1850-1859, Deville 1849, Deville \& Des Murs 1849, Deville \& Sclater 1852, Taczanowski 1884a, 1884b). Especialmente importantes son las colectas de Edward Bartlett en el periodo 18651869 en Chamicuro, Chayaveta (Chyavetas, Chyavitas, Chayavitas), Jeberos (Xeberos), Ucayali, Sarayacu, Nauta, Elvira y los ríos Huallaga y Pastaza (Sclater \& Salvin 1866, Sclater \& Salvin 1873, Taczanowski 1884a, 1884b, 1886) y la extensa colecta ornitológica de John Hauxwell quien aportó especímenes a las colecciones británicas y norteamericanas por casi tres décadas, de las localidades de Chamicuro en el Huallaga, del Napo, Ucayali y en especial de Pebas entre el río Ampiyacu (Ambiyacu) y Amazonas (Taczanowski 1884a, 1884b, 1886, Sclater 1857b, Sclater \& Salvin 1867, Bartlett 1882).

No menos importantes deben considerarse las colectas de Henry Walter Bates en el río Yavarí reportadas entre Perú y Brasil sin una localidad más precisa y que ocurrió en su exploración del Amazonas entre 1848 y 1859, incluyendo colecta de especímenes en Ucayali, Nauta, San Cruz y Pebas (Bates 1863, 1892; Sclater 1857a; Taczanowski 1884a, 1884b); así como la colecta de Henry Whitely, quien próximo a terminar su exploración en Perú en su ruta a Brasil, colectó en las localidades de Balsapuerto, Yurimaguas, Chayavetas, Chamicuro, Nauta, Elvira, Santa Cruz e Iquitos (Yquitos), además por el río Ucayali desde Sarayacu y por el Amazonas hasta Pebas en su viaje hacia Pará (Anónimo 1893; Taczanowski 1884b). A inicios del siglo XX algunas localidades de Loreto fueron visitadas por Malcolm Playfair Anderson y
Wilfred Hudson Osgood del Field Museum of Natural History de Chicago (Anderson 1919) y posteriormente por los hermanos Olalla que colectaron miles de especímenes para el American Museum of Natural History durante varios años en Ecuador, Perú y Brasil (Wiley 2010).

Raimondi y las colecciones nacionales. - El registro en colecciones científicas nacionales alcanza niveles significativos con la llegada a Perú de Antonio Raimondi en 1850, quien, siendo un destacado miembro de la Universidad Nacional Mayor de San Marcos, incluye el ámbito de Loreto en sus famosas expediciones por el territorio peruano. Raimondi reunió una importante colección científica ornitológica que fue albergada por el Museo de Historia Natural de la Universidad Nacional Mayor de San Marcos desde 1921 en un primer ingreso de especímenes (Ridoutt 1941a) y en 1939 con un segundo ingreso después de la muestra del Ministerio de Fomento celebrando el centenario de su nacimiento (La Torre 1991). Todos los ejemplares históricos de la Colección Antonio Raimondi del Museo de Historia Natural de la UNMSM (incluyendo aves de Loreto) son de especial valor, siendo perpetuados en acuarelas encargadas por el director fundador del Museo, el Dr. Carlos Rospigliosi Vigil y fueron presentados en la Exposición de 1926 realizada por el Centenario del nacimiento de Raimondi (La Torre 2012, Fig. 2).

Los viajes de Raimondi a Loreto fueron realizados en 1859 y 1869. En ambos viajes llegó a Yurimaguas y de allí viajó hasta Iquitos, siendo notable que tratara a Loreto como una provincia "Litoral" (Raimondi 1862). En el primer viaje a Loreto llega a Chachapoyas y de allí cursa el Huallaga, Marañón, Ucayali y Amazonas, y también recorre a pie los tramos entre Jeberos y Moyobamba. En el segundo viaje la exploración de Raimondi se extiende más allá de Iquitos por el Amazonas, llegando hasta la frontera con Colombia y Brasil (Raimondi 1874). Antonio Raimondi realizó un activo intercambio científico con Władysław Taczanowski, además Konstanty Jelski lo asistió para su libro "El Perú", y con la compañía del joven Jan Stolzmann, investigador y colector polaco, realizaron colectas en Loreto (Raimondi 1880). Esos ejemplares y los que colectara Stolzmann en su retiro de Perú rumbo a Polonia en 1881, partiendo del Puerto de Belén en Iquitos, permitieron que numerosos ejemplares colectados en Loreto en localidades de Nauta, Iquitos y Pebas fueran citados en el famoso Ornithologie du Pérou (Taczanowski 1884 a, 1884b, 1886).

En la Colección Ornitológica del Museo de Historia Natural de la Universidad Nacional Mayor de San Marcos se conservan doce ejemplares de la Colección Antonio Raimondi, colectados en Loreto, posiblemente entre 1867 y 1876 cuando K. Jelski trabajaba para Raimondi pagado por el Estado peruano. Dos de las especies colectadas son montanas, Nonnula brunnea (MUSMOrn-01720) colectada de Balsapuerto y Eubucco versicolor (MUSM-Orn-35641) de una localidad no precisada; dos especímenes son de la provincia de Alto Amazonas, en el distrito de Jeberos, Tityra semifasciata (MUSMOrn-03573) colectada en Bosque de Jeberos y Pseudo- 
pipra pipra (MUSM-Orn-3527) colectado posiblemente entre el tramo entre Jeberos y Nauta; dos ejemplares fueron colectados en Iquitos, Brotogeris sanctithomae (MUSM-Orn-01780) y Brotogeris versicolurus (MUSMOrn-02013); y seis especímenes fueron colectados en su exploración de Pebas y la frontera con Brasil, Monasa nigrifrons (MUSM-Orn-01687), Euphonia xanthogaster (MUSM-Orn-21428), Cotinga maynana (MUSMOrn-03570), Molothrus oryzivorus (MUSM-Orn-39823),
Piaya melanogaster (MUSM-Orn-36977) y Nyctidromus albicollis (MUSM-Orn-35345). De este grupo solo el ejemplar de Brotogeris sanctithomae (MUSM-Orn-01780) es una piel de estudio, mientras los otros especímenes son aves naturalizadas. Otras dos pieles de estudio, de Euphonia laniirostris (MUSM-Orn-7770) y Vireo olivaceus (MUSM-Orn-18648), presentan como localidad de colecta al distrito de Bajo Biavo que, si bien en la época de su colecta pertenecían a la región Loreto, en la actualidad corresponden a la región de San Martín.
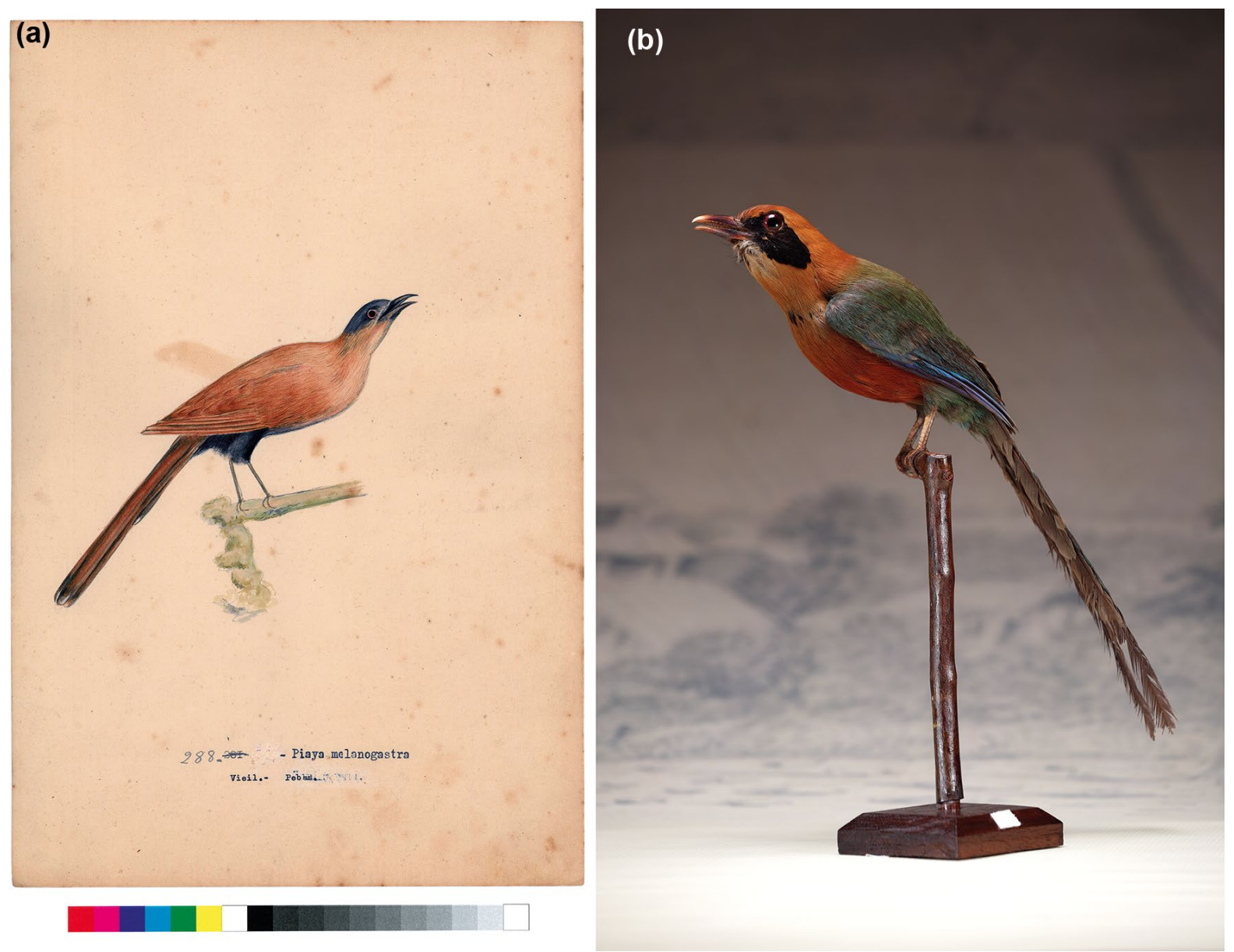

Figura 2. (a) Ilustración de ave recolectada en Loreto y parte de la colección Raimondi enviada a pintar por C. Rospligiosi (Piaya melanogaster), (b) Espécimen naturalizado de la colección Raimondi (Baryphthengus martii).

En la Colección Ornitológica del Museo de Historia Natural de la Universidad Nacional Mayor de San Marcos están bien documentadas las aves de la región de Loreto dentro de sus actuales límites políticos, luego de la separación del departamento de Ucayali en 1980. También están representadas las aves de ámbitos geográficos más extensos que en el momento de su colecta comprendían Loreto, como localidades de San Martín y de Amazonas que se escindieron de Loreto en 1906 y en 1832, respectivamente. Varios de estos especímenes y otros de valor histórico con fecha y colector desconocidos fueron tratados por Ridoutt en los volúmenes 10, 12, 16 y 17 de los Boletines del Museo de Historia Natural UNMSM (Ridoutt 1939, 1940, 1941a, 1941b).
Ornithologie du Pérou. - Las colectas en Loreto fueron la fuente primaria de notables tratados de la avifauna del Perú (Taczanowski 1884a, 1884b, 1886; Zimmer 1924, 1937) y de América (Cory \& Hellmayr 1925, 1927). Vladimir Taczanowskii reunió una de las colecciones más completas del Perú en Europa, a partir del trabajo de colectores europeos y americanos (muchos anteriormente mencionados) y recibiendo un aporte muy significativo a partir del préstamo de los especímenes de la colección Raimondi y de las colectas de Jelski y Stolzmann en el marco de la cooperación científica entre Raimondi y Taczanowskii. Fueron numerosas las especies de aves de Loreto tratadas en los tres volúmenes de Ornithologie du Pérou de Taczanowskii publicados entre 1884 y 1886, desde aves tan grandes como Harpia harpyja y Crax glo- 
bulosa, hasta aves pequeñas como Chloroceryle aenea, Picumnus castelnau y Phaethornis ruber.

Las localidades de colecta de Raimondi con mayor número de especies en Ornithologie du Pérou son Iquitos, Balsapuerto, Pebas, Xeveros, Sarayacu, Ucayali, Yurimaguas y Nauta; mientras que Stolzmann aportó más de 80 especies casi en su totalidad de Yurimaguas y en menor número de Balsapuerto. Por lo menos 430 especies de aves de estos tratados tienen localidades de colecta dentro del ámbito geográfico actual de la región Loreto (Fig. 3).

\section{5. - Myrmelastes plumbeus}

Thamnophilus hyperythrus, Govw, Ann. Mag. N. H., 1855, p. 364 - P. Z. S., 1855 , p. 70 - Sc.. et SALV., P. Z. S., 1866 , p. 185 (Q)

Myrmelastes plumbeus, Sc.., P. Z. S., 1858, p. 274, th. CXLIII

- Cat. Am. B., p. 189 - Scl. et SALv., P. Z. Z., 1866, p. 567 (O').

Thamnophilus plumbeus, Scl. et Salv., P. Z. S., 1873, p. 273.

M. nigricanti plumbeus, alis caudaque obseure nigris; lectricibus alarum apicibus albo guttatis; rostro el pedibus nigris.

Fem. mari similis, sed corpore toto subtus ferrugineo rufo.

ot Ad. - D'un plombé foncé sur tout le corps, un peu moins foncé en dessous qu'en dessus. Ailes et queue noires, à toutes les tectrices alaires terminces par une petite tache blanche, subtriangulaire; sous-alaires schistacées; pli de laile varié de nombreuses taches blanches. Becet pattes noirs. Q D'un plombé bleuatre foncé, uniforme en dessus et sur les cótés de la téte, d'un roux ferrugineux intense en dessous, à sous-caudales d'un brun rougeătre foncé. Ailes noirâtres, à tectrices terminées chacune par une petite tache blanche, triangulaire; sous-alaires d'un gris foncé, tachetié

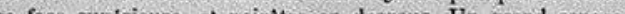
sa face supérieure, et noiratre en dessous. Un grand espace postoculaire dénudè blanchâtre. Bec noiratre; pattes d'un plombé noirátre.

of Longueur de I'aile, 85 ; queue, 53 ; bec, 31 ; tarse, 30 millimètres.

$8 \quad-83 ;-60 ;-26 ;-30$ -

Pelas (HAuxwrLL); Nauta, Xeveros, Santa Cruz (BABTLBTT); Pebas (coll. RAIMONDI); Yquitos (WHITELY).

Figura 3. Descripción de Myrmelastes plumbeus (nombre actual Myrmelastes hyperythrus), a partir de colectas en Loreto realizadas por Hauxwell, Bartlett, Raimondi y Whithely. Extraído del libro Ornithologie du Perou, Vol II, página 24 (Taczanowski 1884b).

Localidades de importancia histórica. - En la historia de la exploración de Loreto fue importante la colecta de aves en localidades en el curso del río Huallaga. En este ámbito fueron especialmente importantes las localidades de Chamicuro, Lagunas y Jeberos en la provincia de Alto Amazonas por la riqueza de especies nuevas para la ciencia descritas en el siglo XIX e inicios de siglo XX (Fig. 4). Desde este acceso a Loreto, fueron importantes Yurimaguas y Balsapuerto como puntos de arribo de los investigadores.

En la ruta del río Ucayali en Loreto fue importante Sarayacu y Cashiboya, el curso del río Marañón y Nauta (Fig. 4). Iquitos no solo fue importante como área de estudio, sino también como zona de aprovisionamiento y descanso en la investigación de aves en el río Napo y del Amazonas en las localidades fronterizas con Brasil y Colombia, en donde Pebas y el río Ampiyacu fueron extensamente explorados en la investigación ornitológica nacional, europea y norteamericana (Fig. 4).

Localidades como Chamicuros, Nauta, Elvira, Lagunas, Santa Cruz, Sarayacu y Orellana entre otras (Fig. 4) fueron visitadas de manera reiterada por varios de estos colectores como otros posteriores y a partir de cuyos especímenes se describieron para la ciencia nuevas especies y subespecies (e.g. Bartlett 1882, Cory 1916, 1919a, 1919b, Chapman 1928, Chubb 1919, 1920, Des Murs 1855, Deville 1849, Deville \& Des Murs 1849, Deville \& Sclater 1852, Gould 1855, Hartert 1900, Hellmayr 1910, Lafresnaye 1851, Sclater 1854, 1855, 1857a, 1857b, 1858, 1860, 1883, Sclater \& Salvin 1866, 1867, 1869, 1881, Sharpe 1885, Taczanowski 1884a, 1884b, Zimmer 1932, 1934, 1936, 1937, 1940, 1947, 1948).

Épocas más recientes. - Hacia el final del siglo XIX, la presencia de investigadores y colectores estadounidenses se hizo más importante en Loreto, destacando las expediciones del Museum of Comparative Zoology de la Universidad de Harvard, del Field Museum of Natural History de Chicago y del American Museum of Natural History de New York. Los especímenes presentes en sus colecciones científicas documentan esta etapa de interés por la Amazonia de Loreto, que se extendió hasta el siglo XX.

Desde mediados del siglo XX se intensificaron los estudios colaborativos con entidades internacionales que exploraron la diversidad de especies de Loreto. El ornitólogo peruano Javier Ortiz de la Puente, quien fuera el primer jefe de la Sección Aves y Mamíferos del Museo de Historia Natural, realizó colectas en Loreto, tanto en 1948 como parte de la "Exploración del río Huallaga" propiciada por UNESCO (Bolívar 1949), como posteriormente entre agosto y setiembre de 1952. En la primera expedición colectó alrededor de cien ejemplares de aves y mamíferos a lo largo del río Huallaga (Bolívar 1949), mientras que, en la segunda visitó Nauta y San Pedro en el Marañón, Alto Tapiche en Requena y el ámbito de la actual reserva nacional Pacaya Samiria creada en 1972 (Fig. 5). En la segunda expedición Javier Ortiz de la Puente viajaba con su reciente esposa y solo dos meses más tarde moriría dramáticamente en Lima; seis ejemplares de esa colecta que incluía trogones, martines pescadores, águilas, loros, mosqueros, semilleros, jacanas, palomas y tiganas, fueron posteriormente identificados y catalogados por John P. O'Neill.

María Koepcke, quien reemplazó a Ortiz de la Puente en la jefatura del departamento de Ornitología del Museo de Historia Natural de la UNMSM, realizó también expediciones a Loreto en 1967, colectando en lo que es hoy la reserva nacional Pacaya-Samiria. Unos años antes, en 1963 , un joven ornitólogo norteamericano con apenas 21 años se apasionó por la avifauna peruana luego de un viaje a la Amazonia de Loreto, era John Patton O’Neill, quien desde ese momento se convierte en el vinculo para el trabajo colaborativo del Museo de Historia Natural de la Universidad Nacional Mayor de San Marcos y la Louisiana State University. El amor por las aves peruanas de O’Neill unió su vida a la Colección Ornitológica del Museo de Historia Natural de la Universidad Nacional Mayor de San Marcos. María Koepcke alentó su trabajo y él durante casi 42 años de investigación en Perú mantuvo una colaboración muy cercana con la colección de aves del Museo, naciendo de este afecto el conseguir financiamiento 


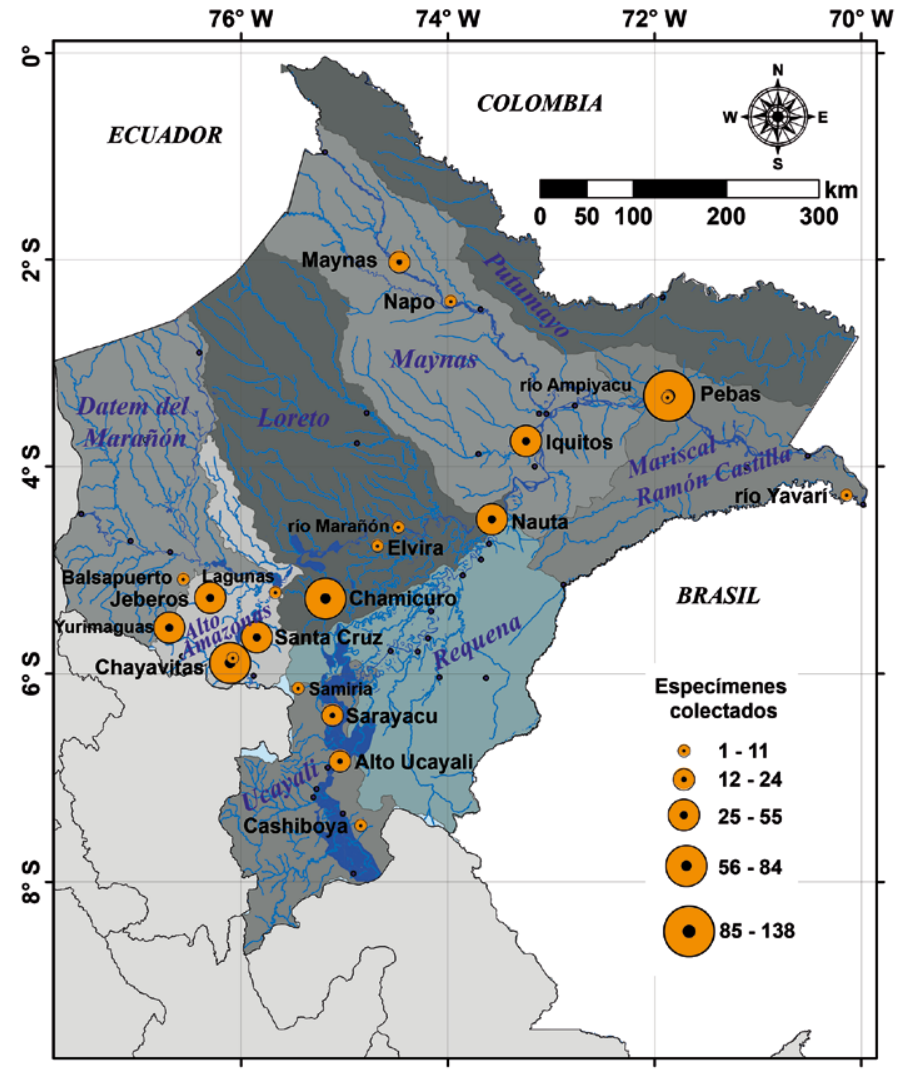

Figura 4. Ubicación de las principales localidades con colectas ornitológicas de valor histórico en las provincias de la región Loreto. El tamaño del símbolo es proporcional al número de especímenes colectados.
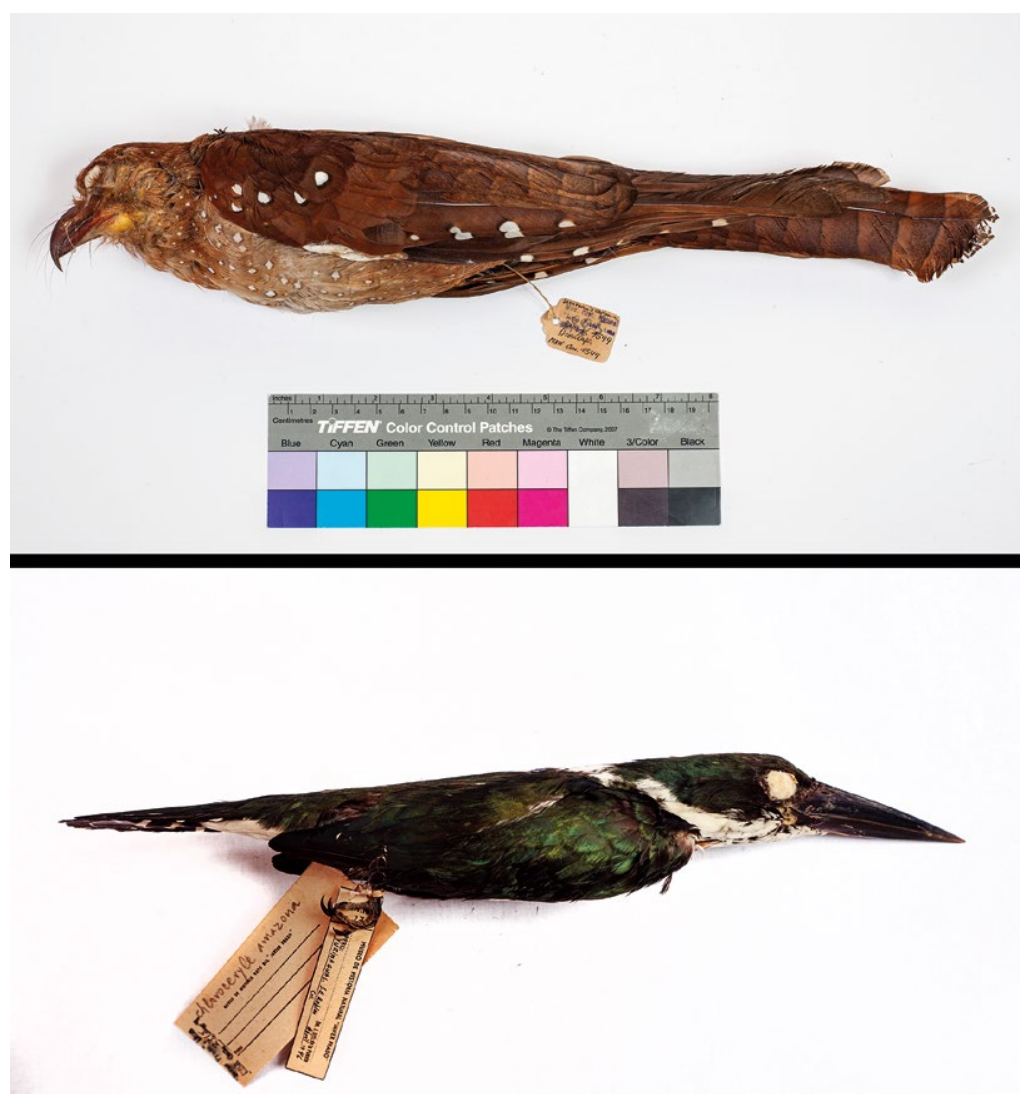

Figura 5. Ejemplares colectados por Javier Ortiz de la Puente en Loreto. (a) Steatornis caripensis del río Huallaga. (b) Chloroceryle amazona de Yurimaguas. 
para la actual edificación que resguarda las colecciones especializadas de aves del Museo de Historia Natural. En la actualidad dos salas con colecciones del departamento de ornitología llevan el nombre de John Patton O’Neill y su legado vive en el corazón de los jóvenes investigadores de esta institución.

En 1996, John P. O’Neill realizó una expedición a Pampa Hermosa, Contamana y río Cushabatay, mientras que el 2000 a Pampa Hermosa y finalmente en mayo de 2005 al lago Rimachi en el distrito de Pastaza, regresando a la que fuera su primera localidad de colecta en Loreto. John P. O'Neill fue el impulsor de mucha de la investigación científica norteamericana en Loreto por casi cuatro décadas. Desde ese entonces los estudios de la avifauna de Loreto se incrementaron tanto en número como complejidad. El desarrollo de las últimas décadas no son objetivo del presente estudio que se enfoca en los estudios y colectas más tempranos. La investigación continua de la avifauna loretana ha hecho posible que esta región sea en la actualidad un punto clave para entender los patrones de diversificación de las aves de la Amazonia.

\section{Literatura citada}

AMNH. 2020. American Museum of Natural History Vertebrate Zoology Collection Database. http://sci-web-001. amnh.org. Acceso 12/2020.

Anderson MB. 1919. Malcolm Playfair Anderson. The Condor 21:115-119.

Anónimo. 1893. Obituary: Henry Whitely. The Ibis. 5(18): 287288.

Bartlett E. 1882. On some Mammals and Birds collected by Mr. J. Hauxwell in Eastern Peru. Proceedings of the Scientific Meetings of the Zoological Society of London. 373-374.

Bates HW. 1863. The naturalist on the river Amazons. 2 Vol. Londres: Murray.

Bates HW. 1892. The Naturalist on the River Amazons, with a memoir of the author by Edward Clodd. Reprint of the Unabridged Edition. With Map and Numerous Illustrations. London: John Murray.

BHL. 2020. Biodiversity Heritage Library. https://www.biodiversitylibrary.org/.

Bolívar C. 1949. International Institute of the Hylean Amazon (IIHA) Report on the Exploration of the River Huallaga, Peru. United Nations Educational, Scientific and Cultural Organization. (https://unesdoc.unesco.org/ ark:/48223/pf0000153782)

Brabourne L, Chubb C. 1917. A List of the Birds of South America. London: R.H. Porter.

Chapman FM. 1928. Mutation in Capito auratus. American Museum Novitates 335:1-21.

Chubb C. 1919. Descriptions of new forms of South and Central American birds. Bulletin of the British Ornithologists' Club 39:51-53.

Chubb C. 1920. Atticora fucata roraimae, and Henicorhina leucosticte hauxwelli subspp. n. described. Bulletin of the British Ornithologists' Club, 40:155-156.

Cory CB. 1916. Descriptions of apparently new South American birds, with notes on some little-known species. Field Museum of Natural History, Ornithological Series
1(10):337-346.

Cory CB. 1919a. Catalogue of birds of the Americas and the adjacent islands in Field Museum of Natural History Part II, no. 2. Field Museum of Natural History Zoological Series 13(203): 317-607.

Cory CB. 1919b. New forms of South American birds and proposed new subgenera. Auk 36:273-276.

Cory CB, Hellmayr CE. 1925. Catalogue of birds of the Americas and the adjacent islands in Field Museum of Natural History Part IV. Field Museum of Natural History Zoological Series 13(224):1-390.

Cory CB, Hellmayr CE. 1927. Catalogue of birds of the Americas and the adjacent islands in Field Museum of Natural History Part 5. Field Museum of Natural History Zoological Series 13(242):1-517.

De Castelnau F. 1850-1859. Expédition dans les parties centrales de l'Amérique du Sud, de Rio de Janeiro à Lima, et de Lima au Para; exécutée par ordre du Gouvernement Français pendant les années 1843 à 1847. Paris: Chez P. Bertrand.

Des Murs 0. 1855. Oiseaux. In: Animaux nouveaux ou rares recueillis pendant l'expédition dans les parties centrales de l'Amérique du Sud, de Rio de Janeiro à Lima, et de Lima au Para. P Bertrand, Paris. Pp. 1-98.

Deville E. 1849. Description de quelques Mammifères et Oiseaux nouveaux de l'Amérique méridionale. Revue et Magasin de Zoologie pure et appliquée S.2, T.1:55-58.

Deville E, Des Murs 0. 1849. Note sur une nouvelle espèce de Couroucou (Trogon ramoniana, et sur le Trogon meridionalis. Revue et Magasin de Zoologie pure et appliquée S.2, T.1:331-333.

Deville E, Sclater PL. 1852. Description d'une nouvelle espèce de Cotinga provenant de l'expédition de MM. de Castelnau et Deville dans l'Amérique du Sud. Revue et Magasin de Zoologie pure et appliquée S.2, T.4: 226-227.

Espinoza Soriano, W. 2007. Amazonia del Perú. Historia de la gobernación y comandancia general de Maynas (hoy regiones de Loreto, San Martín, Ucayali y provincia de Condorcanqui). Del siglo XV a la primera mitad del siglo XIX. Lima: Fondo Editorial del Congreso del Perú, Banco Central de Reserva del Perú, PromPerú.

GBIF.org. 2020. GBIF Home Page. Available from: https://www. gbif.org [13 diciembre 2020].

Gould J. 1855. Descriptions of eight new species of birds from South America. Annals and Magazine of Natural History, London 15:343-346.

Hartert E. 1900. Allgemeines und Specielles über Kolibris. Journal für Ornithologie 48: 350-368.

Hellmayr CE. 1910. Notes sur quelques oiseaux de l'Amérique tropicale. Revue Francaise d'Ornithologie Scientifique et Pratique 1:161-165.

Hastert E. 1918. Types of birds in the Tring Museum. Novitates Zoologicae 25:4-63.

iDigBio. 2020. Integrated Digitized Biocollections. http://www. idigbio.org. Acceso 12/2020.

Lafresnaye F. 1851. Essai d'une monographie du genre Picucule (Buffon), Dendrocolaptes (Hermann, Illiger), devenu aujourd'hui la sous-famille Dendrocolaptinae (Gray, Genera of birds), de la famille Certhiadæ de Swains. Revue et Magasin de Zoologie pure et appliquée S.2, T.3: $145-147$.

La Torre R. 1991. Percances del legado de Antonio Raimondi. 
Boletín de la Sociedad Peruana de Medicina Interna. $4(3)$.

La Torre R. 2012. Estudio Introductorio. En: El Perú Tomo I. Parte Preliminar. Edición Ilustrada. Lima: Fondo Editorial Universidad Tecnológica del Perú.

León Pinelo A. 1943. El Paraíso en el Nuevo Mundo (Edición de Raúl Porras Barrenechea). Lima: Comité del IV centenario del descubrimiento del Amazonas.

Lecroy M. 2005. Type specimens of birds in the American museum of natural history part 6. Passeriformes: Prunellidae, Turdidae, Orthonychidae, Timaliidae, Paradoxornithidae, Picathartidae and Polioptilidae. Bull Am Museum Nat Hist. 292:132. doi:10.1206/724.1.

Lecroy M. 2012. Type Specimens of Birds in the American Museum of Natural History Part 10. Passeriformes: Emberizidae: Emberizinae, Catamblyrhynchinae, Cardinalinae, Thraupinae, and Tersininae. Bull Am Museum Nat Hist. 368:125.

LeCroy M, Sloss R. 2000. Type specimens of birds in the American Museum of Natural History part 3. Passeriformes: Eurylaimidae, Dendrocolaptidae, Furnariidae, Formicariidae, Conopophagidae, and Rhinocryptidae. Bull Am Museum Nat Hist.(257):88.

Macera P, Jiménez Borja A, Franke I. 1997. Trujillo del Perú, Baltazar Jaime Martínez Compañón, Acuarelas Siglo XVIII. Lima: Fundación del Banco Continental.

Mlíkovský J. 2009. Types of birds in the collections of the Museum and Institute of Zoology, Polish Academy of Sciences, Warszawa, Poland. Part 4: Varia, addenda and conclusions. J Natl Museum (Prague), Nat Hist Ser. 178(5):17-180.

NMNH. 2020. Smithsonian National Museum of Natural History Collection Records. http://collections.nmnh.si.edu. Acceso 12/2020.

Paz Soldán MF. 1865. Atlas Geográfico del Perú. París: Librería de Augusto Durand.

Pons Muzzo G. 1962. Las fronteras del Perú: Estudio histórico. Lima: Talleres Gráficos Iberia, S. A.

Raimondi GA. 1862. Apuntes sobre la provincia litoral de Loreto, Tipografía Nacional. Lima: Imp. Manuel D. Cortés.

Raimondi GA. 1874. El Perú. Parte Preliminar (Tomo I). Lima: Imprenta del Estado.

Raimondi GA. 1880. El Perú. Historia de la Geografía del Perú (Tomo III). Lima: Imprenta del Estado.

Ridoutt CA. 1939. Ornitología del Perú. Boletín del Museo de Historia Natural 3(10):71-73.

Ridoutt CA. 1940. Ornitología del Perú. Boletín del Museo de Historia Natural 4(12):94-95.

Ridoutt CA. 1941a. La Colección Ornitológica del Museo. Boletín del Museo de Historia Natural 5(16):91-108.

Ridoutt CA. 1941b. La Colección Ornitológica del Museo. Boletín del Museo de Historia Natural 5(17):248-263.

Roselaar CS. 2003. An inventory of major European bird collections. Bull Ornithol Club. 123:253-337.

Sclater PL. 1854. Descriptions of six new species of birds of the subfamily Formicarinae. Proceedings of the Zoological Society of London 22(1): 253-255.

Sclater PL. 1855. A draft arrangement of the genus Thamnophilus, Vieillot. Edinburgh New Philosophical Journal 1: 226-249.

Sclater PL. 1857a. Characters of some apparently New Spe- cies of American Ant-Thrushes. Proceedings of the Zoological Society of London. 25: 46-48. https://doi. org/10.1111/j.1096-3642.1857.tb01195.x

Sclater PL. 1857b. On a collection of birds transmitted by Mr. H. W. Bates from the Upper Amazon. Proceedings of the Zoological Society of London 25:261-268. https://doi. org/10.1111/j.1096-3642.1857.tb01238.x.

Sclater PL. 1858. Synopsis of the American Ant-birds (Formicariidae). Part II. containing the Formicivorinae or Ant-Wrens. Proceedings of the Zoological Society of London 1858: 232-254.

Sclater PL. 1860. Characters of ten new species of American birds. Proceedings of the Zoological Society of London 28(3):461-467.

Sclater PL. 1883. Descriptions of five apparently new species of South American Passeres. Proceedings of the Scientific Meetings of the Zoological Society of London. London: Messrs. Longmans, Green, and Co. Pp. 653-654.

Sclater PL, Salvin O. 1866. Catalogue of birds collected by Mr. E. Bartlett on the river Ucayali, Eastern Peru, with notes and descriptions of new species. Proceedings of the Zoological Society of London 2: 175-201.

Sclater PL, \& Salvin O. 1867. List of birds collected at Pebas, upper Amazons, by Mr. John Hauxwell, with notes and descriptions of new species. Proceedings of the Zoological Society of London 1867:977-981.

Sclater PL, Salvin 0.1869. On two new birds collected by Mr. E. Bartlett in Eastern Peru. Proc. Zool. Soc. London 1869: 437-439.

Sclater PL, Salvin 0. 1873. On the birds of eastern Peru, with notes on the habits of the birds by Edward Bartlett. Proceedings of the Zoological Society of London 1873:252-311.

Sclater PL, Salvin 0. 1881. Descriptions of some new species of South-American Birds of the families Tyrannidae and Formicariidae. Ibis (5): 267-271.

Scott HV. 2010. Paradise in the New World: an Iberian vision of tropicality. Cultural Geographies 17(1):77-101. doi: $10.1177 / 1474474009350000$.

Sharpe RB. 1874. Catalogue of the birds in the British Museum. Catalogue of the Accipitres, or Diurnal Birds of Prey, in the Collection of the British Museum. London: Taylor and Francis.

Sharpe RB. 1885. Catalogue of the birds in the British Museum. Catalogue of the Passeriformes, or perching birds, in the collection of the British Museum. London: Taylor and Francis.

Spix JB von. 1824. Avium species novae, quas in itinere per Brasiliam annis MDCCCXVII-MDCCCXX jussu et auspiciis Maximiliani Josephi I. Bavariae regis augustissimi. Suscepto. Collegit et descripsit Dr. Joannes Bapt. de Spix. Monachii (Múnich): Impensis Editoris.

Taczanowski L. 1884a. Ornithologie du Pérou. Vol. 1. Rennes: Typographie Oberthur.

Taczanowski L. 1884b. Ornithologie du Pérou. Vol. 2. Rennes: Typographie Oberthur.

Taczanowski L. 1886. Ornithologie du Pérou. Vol. 3. Rennes: Typographie Oberthur.

Tschudi JJ von. 1844-1846a. Untersuchungen über die Fauna Peruana. St. Gallen: Druck und Verlag von Scheitlin und Zollikofer.

Tschudi JJ von. 1966. Testimonio del Perú 1838-1842. Lima: 
Consejo Económico Consultivo Suiza-Perú.

van den Hoek Ostende L, Dekker R, Keijl G. 1997. Type-specimens of birds in the National Museum of Natural History, Leiden Part 1. Non-Passerines. NNM Tech Bull. 1(30.xii):1-248.

VertNet. 2020. VertNet. http://www.vertnet.org. Acceso $12 / 2020$.

Wiley RH. 2010. Alfonso Olalla and his family: the Ornithological Exploration of Amazonian Peru. Bulletin of the American Museum of Natural History 343:1-68.

Zimmer JT. 1924. Studies on Peruvian Birds. $N^{\circ} 40$. Notes on the Genus Veniliornis. American Museum Novitates 1159:1-4.

Zimmer JT. 1932. Studies of Peruvian birds. $\mathrm{N}^{\circ} 05$. The genera Herpsilochmus, Microrhopias, Formicivora, Hypocnemis, Hypocnemoides, and Myrmochanes. American Museum Novitates 538: 1-27.

Zimmer JT. 1934. Studies of Peruvian birds. $N^{\circ} 15$. Notes on the genus Xiphorhynchus. American Museum Novitates 756:1-20.

Zimmer JT. 1936. Studies of Peruvian birds. $\mathrm{N}^{\circ} 22$. Notes on the Pipridae. American Museum Novitates 889:1-29.

Zimmer JT. 1937. Studies on Peruvian Birds $\mathrm{N}^{\circ} 27$. Notes on the genera Muscivora, Tyrannus, Empidonomus, and Sirystes, with further notes on Knipolegus. American Museum Novitates 962:1-28

Zimmer JT. 1940. Studies of Peruvian birds. $N^{\circ} 34$. The genera Todirostrum, Euscarthmornis, Snethlagea, Poecilotriccus, Lophotriccus, Myiornis, Pseudotriccus and Hemitriccus. American Museum Novitates 1066: 1-23.

Zimmer JT. 1947. Studies of Peruvian birds. $\mathrm{N}^{\circ} 51$. The genera Chlorothraupis, Creurgops, Eucometis, Trichothraupis, Nemosia, Hemithraupis, and Thlypopsis, with additional notes on Piranga. American Museum Novitates 1345:1-23.

Zimmer JT. 1948. Studies of Peruvian birds. $\mathrm{N}^{\circ} 53$. The family Trogonidae. American Museum Novitates 1380: 1-56.

\section{Agradecimientos / Acknowledgments:}

Agradecemos a Wildlife Conservation Society - Perú por el soporte para el presente estudio. Gracias a Daniel Cossios por la invitación para este número especial. Agradecernos a Mario Pezua, Mónica Paredes, Daniel Muñoz y Juan Francisco Esteves por la ayuda en la recopilación de información de las bases de datos de colecciones científicas.

\section{Conflicto de intereses / Competing interests:}

CA es editor de la RPB, no participó en el el proceso de edición. Los autores no incurren en conflictos de intereses.

\section{Rol de los autores / Authors Roles:}

LS, AA y CA Conceptualización, Investigación, Escritura-Preparación del borrador original, Redacción-revisión y edición.

Fuentes de financiamiento / Funding:

Wildlife Conservation Society - Perú, VRIP-UNMSM (Proyecto 161001161).

\section{Aspectos éticos / legales; Ethics / legals:}

Los autores declaran no haber incurrido en ningún aspecto antiético ni omitido norma legal nacional o internacional en la investigación y elaboración del articulo. 
